\title{
TBM
}

\section{Implementation of an evidence-based intervention to promote colorectal cancer screening in community organizations: a cluster randomized trial}

\author{
Annette E. Maxwell, DrPH ${ }^{1,2}$ Leda L. Danao, PhD, ${ }^{1}$ Reggie T. Cayetano, MD, MPH, ${ }^{1}$ Catherine M. Crespi, PhD, ${ }^{1}$ \\ Roshan Bastani, $\mathrm{PhD}^{1}$
}

Fielding School of Public Health and Jonsson Comprehensive Cancer Center, UCLA Kaiser Permanente Center for Health Equity, University of California, Los Angeles, CA, USA

${ }^{2} 650$ Charles Young Dr. South, A2125 CHS, Box 956900, Los Angeles, CA 90095-6900, USA

Correspondence to: A Maxwell amaxwell@ucla.edu

Cite this as: TBM2016;6:295-305 doi: 10.1007/s13142-015-0349-5

\section{Abstract}

The implementation of evidence-based strategies to promote colorectal cancer (CRC) screening remains challenging. The aim of this study is to evaluate two strategies to implement an evidence-based intervention to promote CRC screening in Filipino American community organizations. Twenty-two community organizations were randomized to either a basic or enhanced implementation strategy. In both arms, community health advisors recruited participants non-adherent to CRC screening guidelines, conducted educational sessions, distributed print materials and free fecal occult blood test kits, reminded participants to get screened, and mailed letters to participants' providers. In the enhanced arm, leaders of the organizations participated in implementation efforts. While the effectiveness was similar in both arms of the study (screening rate at 6-month follow-up was $53 \%$ in the enhanced arm, $49 \%$ in the basic arm), 223 participants were screened in the enhanced arm versus 122 in the basic arm. The enhanced implementation strategy reached $83 \%$ more participants and achieved a higher public health impact.

Trial registration: NCT01351220 (ClinicalTrials.gov)

\section{Keywords}

Implementation, Lay health workers, RE-AIM evaluation framework, Filipino Americans, Evidencebased intervention, Colorectal cancer screening

\section{BACKGROUND}

Evidence-based interventions to promote cancer screening need to be widely implemented to improve population outcomes, yet much remains to be learned about best implementation strategies in real-world settings [1]. This paper reports data from an implementation trial that compared two alternative strategies to implement a multi-component evidence-based intervention to promote colorectal cancer (CRC) screening in Filipino American community organizations. In contrast to efficacy and effectiveness trials that focus primarily on the assessment of behavioral or health outcomes, this trial assessed outcomes

\section{Implications}

Practice: Community-based organizations are able to implement evidence-based strategies to promote colorectal cancer screening among their members, if they receive technical and financial support.

Policy: Health promotion outreach in non-clinical settings can reach underserved groups, including those who do not have health insurance.

Research: Future studies should examine what resources would be required for community organizations to sustain cancer screening and other health promotion programs.

Electronic supplementary material

The online version of this article (doi:10.1007/ s13142-015-0349-5) contains supplementary material, which is available to authorized users. pertaining to the reach of the intervention, its effectiveness, and its implementation.

Filipino Americans are the second largest US Asian group after Chinese Americans [2] and have low rates of colorectal cancer (CRC) screening [3, 4] and poor 5 -year survival after CRC [5]. To address this disparity, evidence-based interventions to promote CRC screening are needed in this community. In prior work (CRC1 Study), we developed and tested a multicomponent intervention to promote CRC screening among Filipino Americans and demonstrated its effectiveness in a randomized trial. The intervention we tested consisted of a small-group educational session at community venues, distribution of print materials and free fecal occult blood test (FOBT) kits, and reminder calls to participants and letters to participants' providers, asking them to recommend CRC screening for their patients [6]. Many of these intervention components are recommended for dissemination by the Community Guide to Preventive Services [7]. 
The current trial (CRC2 Study) focused on the implementation of the previously developed evidencebased intervention in the Filipino American community to increase CRC screening. The terms "implementation" and "dissemination" are often used interchangeably, and the lack of standardized terminology has been noted [8]. Both implementation and dissemination research focus on moving evidence-based interventions to end-users. However, dissemination often is focused on making the potential adopters aware of an intervention, while implementation refers to more hands-on strategies for putting programs into place [9]. Based on this definition, we describe our study as an implementation trial. Many prior trials to promote CRC screening have been conducted in clinical settings [10-17]. While a few pilot studies have demonstrated the feasibility of promoting CRC screening in community settings such as churches [18-20], few large trials have been conducted in these venues [21, 22]. This study reports on a large CRC screening controlled trial conducted in partnership with a variety of community organizations.

\section{METHODS}

The goal of the study was to assess the impact of two strategies-a basic and an enhanced strategy-to implement the intervention within community organizations and to increase CRC screening among Filipino Americans. Similar to other community-based health promotion programs [18, 23-25], community health advisors (CHAs) implemented all program components. Guided by the Racial and Ethnic Approaches to Community Health (REACH) 2010 Model of Change [26], we aimed to increase community awareness of the importance of CRC screening and to develop needed community capacity by training CHAs on how to promote CRC screening in their organizations. In one arm of the study (enhanced implementation), we also engaged the organizations' leaders and assisted them in planning activities to support the promotion of CRC screening. Because the CHAs and community leaders are part of the Filipino American community, they can interact with community members and serve as change agents, even for individuals who do not see a health care provider on a regular basis.

Recruitment and eligibility of organizations-We recruited Filipino American community organizations by approaching organizations that had participated in our previous study promoting CRC screening (previously exposed to intervention), other faith-based organizations in Los Angeles and Orange County cities and neighborhoods with large Filipino American populations that were identified online, other nonfaith-based organizations that were drawn from a Filipino Consumer Guide, and organizations that were referred to us by community partners. A total of 44 organizations completed a brief survey to assess eligibility (membership of at least 150 Filipino Americans age 50 and over and interest in promoting CRC screening among their members). Stipends were offered to eligible organizations and to CHAs for attending trainings, including human subjects research training, and for completing research tasks related to subject recruitment and tracking of their activities regarding intervention implementation. Details of the recruitment process and yields have been previously reported [27]. We stopped recruitment when we had identified 22 community organizations that agreed to adopt the CRC screening promotion program and were able to name five CHAs.

Randomization of organizations-Since the trial involved relatively few organizations, restricted randomization [28] was used to ensure balance and to avoid contamination across arms. We enumerated all twogroup equal allocations that balanced the arms on faith-based versus non-faith-based and new versus previously exposed organizations, and zip code-level mean income and education, and also kept three organizations that were in close geographic proximity in the same arm, and randomly selected one of these allocations. By flipping a coin, the two groups were randomly assigned to the basic or the enhanced implementation strategy.

Basic and enhanced implementation strategies-CHAs in both arms of the study received an initial 6-h training that included information on recommended CRC screening tests and study protocols and materials for recruiting participants, assessing eligibility, and administering the CRC promotion program, followed by a 1-2-h review session after 1-2 months [29]. CHAs were instructed to ask participants to submit completed FOBT kits to their provider or to request a CRC screening test from their provider. CHAs were informed that they could refer participants without health insurance to a specific clinic that had agreed to evaluate completed FOBT kits, inform patients about the test results, and charge the cost for their services to the study. Each CHA received a binder with study materials. To ensure correct implementation of the educational session, research staff observed the first CHA-led small group educational session at each organization and provided support and feedback. Other CHAs of the same organization were encouraged to observe this session. These initial training sessions were held at individual organizations between January and October 2011.

Organizations in the enhanced implementation arm received three additional components to build their capacity to implement the CRC screening promotion program:

- CHA booster sessions at 2, 4, and 6 months after the first educational session, in which research staff conducted site visits to discuss study activities with the CHAs, answer questions, and troubleshoot problems with recruitment or with administering intervention components;

- At each organization, a workshop was conducted with two leaders (e.g., pastor, director), two CHAs, and two active members to facilitate the 
organization's goal setting and development of a plan of action to increase awareness of the burden of colorectal cancer in the Filipino American community, advocate for prevention and early detection among its members, and support the CHAs in increasing colorectal cancer screening among Filipino Americans. Each organization was asked to implement six additional activities to promote CRC screening among members such as designating $\mathrm{CRC}$ screening promotion as a priority project of the $\mathrm{CBO}$, dedicating at least one event such as a fundraiser dance to enhance community awareness of the project, honoring the CHAs during a public event, collaborating with other organizations in promoting CRC screening, or celebrating National CRC Awareness Month. These workshops were conducted in November and December 2011, which was 4 to 7 months after the initial training session for most organizations.

- One leader per organization joined the Community Advisory Board that met six times during the study to provide advice regarding study activities and to discuss potential activities to promote CRC screening, and to report successes, barriers, and facilitators of CRC screening promotion and lessons learned back to the group.

Recruitment and eligibility of participants-Each CHA was asked to recruit up to ten eligible participants using flyers and their personal and organizational networks. To assess eligibility, CHAs administered a short screening questionnaire. Filipino Americans between the ages of 50 and 75 years with no history of CRC who were not adherent to $\mathrm{CRC}$ screening guidelines (no FOBT in the past 12 months and no sigmoidoscopy in the past 5 years and no colonoscopy in the past 10 years) were eligible to participate.

Implementation of the evidence-based program to promote CRC screening-CHAs conducted 45-min educational sessions with Filipino Americans to discuss and encourage CRC screening, distributed print information and FOBT kits to participants, urged participants to discuss CRC screening with their provider, and to return the completed FOBT to their provider or to schedule an endoscopy; see detailed description in [6]. They reminded participants to obtain CRC screening in subsequent encounters either face to face or by telephone. If participants provided contact information of their provider, CHAs mailed a letter to the provider to inform them that their patient had received information on CRC screening tests and to encourage physicians to offer CRC screening. During the trial, some CHAs made modifications to the study protocol, such as referring participants to the free clinic even if they had health insurance and conducting educational sessions with individuals rather than with small groups. We did not interfere with these modifications and captured them in our assessments, as recommended for implementation research [30, 31].

Assessments-After obtaining informed consent, CHAs administered a baseline survey to participants to assess knowledge, attitudes, and beliefs regarding CRC screening, access to health care, and demographic characteristics. Implementation of study activities and implementation outcomes were assessed through log sheets in which CHAs noted completed activities and through 6-month follow-up telephone surveys of participants that were conducted by members of the research team who were not involved in intervention implementation. Participants received a \$20 incentive to complete the baseline survey and a $\$ 25$ incentive to complete the 6-month follow-up survey.

All intervention activities and assessments were approved by the University of California Los Angeles Human Subjects Committee.

Outcome measures-We developed metrics consistent with the Reach, Effectiveness, Adoption, Implementation and Maintenance (RE-AIM) framework of Glasgow and colleagues [32, 33] to evaluate the two implementation strategies. The analysis for this paper examines Implementation of the program by target organizations and staff, Reach of the program into the target population, and Effectiveness among those individuals. Adoption, which in our study refers to the number of organizations and number of CHAs who agreed to implement the program, was established by the design of the trial (11 organizations agreeing to implement the program were allocated to each arm; 5 CHAs were trained per organization) and not considered an outcome. We have little information on Maintenance due to the limited study period.

The specific outcome measures were assessed at three levels (see Table 3):

1. Organizational-level implementation, defined as the proportion of organizations who implemented the program by promoting CRC screening;

2. CHA-level implementation, defined as the proportion of trained CHAs who delivered the program to one or more individuals; $C H A$-level reach, defined as the number of individuals reached per implementing CHA; 3. Individual-level effectiveness, defined as the proportion of enrolled individuals who obtained CRC screening.

In addition, we assessed reach per organization, defined as the number of individuals who received the program (i.e., who enrolled in the study) per organization; and impact per organization, defined as the number of individuals obtaining CRC screening per organization. We also tabulated total reach and total impact, which summed across all organizations.

Statistical analysis-Baseline characteristics of organizations in the two arms were compared using Fisher exact and Mann-Whitney tests. Baseline characteristics of participants were compared between arms using chi-square and two-sample $t$ tests.

Statistical tests comparing organizational adoption and CHA adoption were not conducted since these metrics were similar by design. We compared organizational implementation between arms using a Fisher exact test. CHA implementation was compared using a logistic mixed effects model with CHA as unit of analysis 
and CHA delivered program to one or more individuals (yes/no) as dependent variable. CHA reach was compared using a linear mixed effects model with CHA as unit of analysis and number of enrolled participants per CHA as dependent variable. Both of these models included arm and stratification variables (faith versus non-faith-based organization, new versus previously exposed to $\mathrm{CRC} 1$ ) as independent variables and random intercepts for organization. Effectiveness was compared using a logistic mixed effects model with participant self-reported screening (yes/no) as dependent variable, arm and stratification variables as independent variables, and random intercepts for organization; we assumed individuals not contacted at follow-up were not screened for CRC, and we controlled for length of US residency, education, and health insurance status. The latter two control variables were included to help adjust for income and membership in recruiting organization, which were imbalanced across arms but also had a substantial number of missing values. Reach per organization was compared using linear regression with organization as unit of analysis and number reached per organization as dependent variable, and controlled for the stratification variables. Impact per organization was compared using the same approach with number of individuals screened per organization as dependent variable.

We also computed standardized effect sizes (difference in mean divided by standard deviation) for continuous variables and $h$ effect sizes to compare proportions. The $h$ effect sizes used the arcsine transformation method of Cohen [34]. For both effect size measures, $0.2,0.5$, and 0.8 are considered small, medium, and large, respectively [34]. The study was powered to detect a difference in screening rates (effectiveness) of 10-15 percentage points, after adjusting for clustering [35].

\section{RESULTS}

Over a 3-year period (January 2011 to March 2014), substantial efforts and resources went into building the capacity of the CHAs and the organizations to promote CRC screening. Two research assistants provided more than 70 training and booster sessions to CHAs and 17 trainings and follow-up strategic planning sessions to organization leaders. They attended initial group sessions at 16 organizations and made numerous site visits to administer assessments, pick up research materials, or attend community events. They facilitated six community advisory board meetings that involved multiple phone calls prior to each meeting with community partners to jointly plan the agenda. The number of contacts varied for the two arms of the study.

Consort flow diagram-Of 44 organizations that met eligibility criteria, 22 organizations $(50 \%)$ agreed to participate in the study and were randomized. As shown in Fig. 1, 7 organizations and 30 CHAs recruited and delivered the program to 260 participants in the basic arm and 10 organizations and 40 CHAs recruited and delivered the program to 440 participants in the enhanced arm. Five out of 22 organizations ( 4 in the basic arm and 1 in the enhanced arm) and a total of 43 out of 113 trained CHAs did not implement the program. Eighty-one percent of eligible participants completed 6-month follow-up surveys. The majority of participants who did not complete the follow-up survey could not be reached by phone. A total of 27 participants were excluded from the analysis because they were found to be baseline adherent to $\mathrm{CRC}$ screening $(N=9)$, did not meet age eligibility criteria $(N=9)$, were enrolled in both arms of the study $(N=4)$, or became CHAs $(N=5)$.

Implementation-CHAs administered educational sessions to 673 eligible participants during which they passed out FOBT kits and print materials. They conducted a total of 250 sessions and $47 \%$ of these were only attended by one person (43/90 sessions, $48 \%$ in the basic arm; 75/160 sessions, $47 \%$ in the enhanced arm). Based on $\log$ sheets, CHAs referred $56 \%$ of participants to low-cost or no-cost clinics, issued reminders to $52 \%$ of participants, and sent letters to physicians to $61 \%$ of participants who provided physician information (33\% of the total sample). There was very high agreement on CHA and participant reports of implementation on reported delivery and receipt of educational sessions (99\%) and FOBT kits (95\%). Of participants who completed the 6-month follow-up survey, $96 \%$ reported that the CHA had recommended CRC screening and $80 \%$ reported that the CHA had advised them to discuss CRC screening with their physician. Implementation of intervention components was not significantly different between the two arms of the study.

Baseline characteristics of organizations and participantsAs shown in Table 1, 10 of the 17 organizations that implemented the program were faith-based and 10 had previously been exposed to our CRC1 study. The organizations had been in operation for an average of 39 years, offered on average two health-related programs prior to participation in our study, and had between 150 and 15,300 members, with no significant differences between the two arms.

As shown in Table 2, study participants were on average 61 years of age and had lived in the USA for 17 years, on average. Most were female and married/ living together, and $55 \%$ reported an annual household income under $\$ 20,000$. Almost all were born in the Philippines, but only $22 \%$ used mostly Filipino when speaking with friends. Participants in the basic arm of the study had lived about 3 years longer in the USA than those in the enhanced arm. Participants in the basic arm were also significantly more likely to report a higher annual household income than those in the enhanced arm. Compared to a sample of Filipino Americans who participated in the populationbased California Health Interview Survey and met 


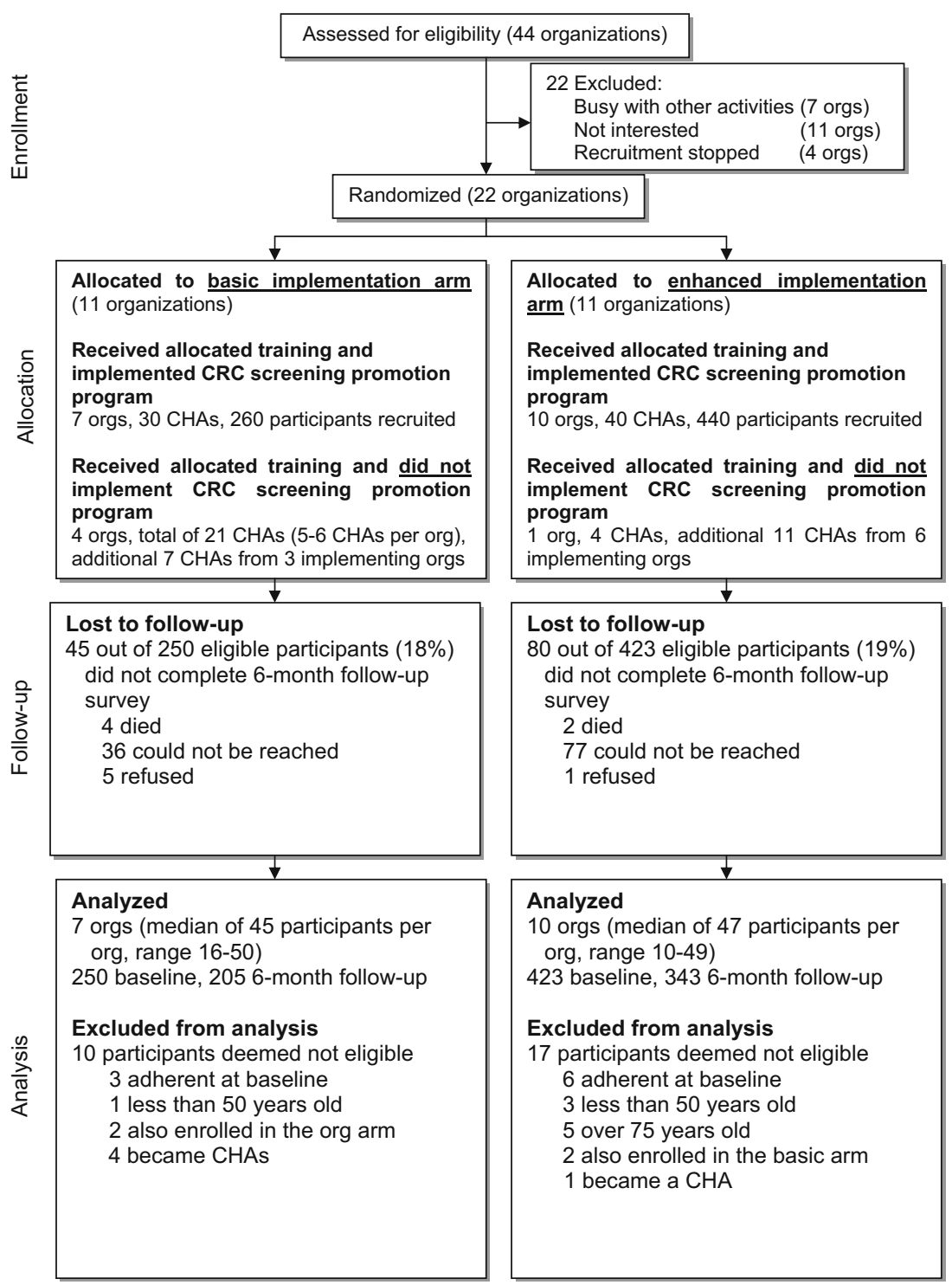

Fig. 1 | Consort flow diagram for implementation trial to promote colorectal cancer screening through Filipino American community organizations

eligibility criteria for our study (age 50-75, nonadherent to CRC screening guidelines; see last column in Table 1), study participants were similar to the California population-based sample with respect to age, gender, length of residency in the USA, and marital status. However, our study sample was more likely to be born in the Philippines and more likely to use mostly Filipino with friends, and less likely to have health insurance. Of those who reported their annual household income in our sample ( $86 \%), 55 \%$ reported an income under $\$ 20,000$, compared to only $17 \%$ in the population-based California sample. Despite the very low income, our study sample included more college graduates than the California sample. The proportion of females in our sample is similar to the proportion in 2010 United States Census data for
Filipino Americans (57\% female in the 35-64 age group; $62 \%$ in the $\geq 65$ age group, data not shown).

Performance of basic and enhanced implementation strategy-As shown in Table 3, several outcome measures were higher in the enhanced arm than in the basic arm; however, no differences were statistically significant. At the level of the organization, the proportion of organizations that implemented the program was higher in the enhanced arm of the study $(91 \%)$ than in the basic arm $(64 \%)$, which constitutes a medium to large effect size. At the level of the CHAs, the proportion of trained CHAs who delivered the program was similar in both arms of the study $(78-81 \%)$, but the enhanced arm had a higher number of active CHAs who recruited participants and delivered the program (40 vs. 30$)$. This in turn led to the enhanced arm 
reaching more individuals (38 \pm 17 per organization versus $23 \pm 21$ per organization in the basic arm), which constitutes a large effect size and almost reaches statistical significance $(P=0.06)$. At the level of the individual participants, based on intent-to-treat analysis with single imputation of unscreened for missing values, the effectiveness of the program was similar in the two arms (53\% of enrolled participants received screening in the enhanced arm versus $49 \%$ in the basic arm). However, due to the higher reach in the enhanced arm, there was a higher impact as well, with an average of 20 individuals screened per organization in the enhanced arm compared to an average of 11 in the basic arm. This constitutes a medium to large effect size.

A total of 345 participants reported receipt of a CRC screening test at 6-month follow-up; 305 completed an FOBT only and 40 received another CRC screening test. Due to the substantially higher reach in the enhanced arm of the study, the impact of the program (number of participants $\times$ screening rate) was also substantially higher in the enhanced arm (223 participants screened) than in the basic arm (122 participants screened), an $83 \%$ difference.

We were able to verify self-reported CRC screening for 177 participants $(51 \%$ of the participants who selfreported CRC screening at 6-month follow-up in the basic arm and $52 \%$ of those who self-reported screening in the enhanced arm). These participants had their FOBT evaluated at the aforementioned clinic that charged the study for FOBT processing. Agreement between self-reported CRC screening and clinic validated screening was $84 \%$ in the basic arm and $94 \%$ in the enhanced arm. We were not able to verify selfreport for participants who had a CRC test at another clinic or provider or for participants who reported that they did not get screened during the follow-up period. Figure 2 visually summarizes the performance of the two implementation strategies on Implementation, Effectiveness, Reach, and the summary Impact dimensions. Despite similar effectiveness of both strategies, implementation by more organizations in the enhanced implementation arm increased the reach and impact of the program substantially.

\section{DISCUSSION}

We assisted community organizations to implement an evidence-based program to promote CRC screening in the Filipino community in the Los Angeles area. The majority of the organizations that had agreed to participate in the study were able to implement the program components. Two implementation strategies, a basic and an enhanced strategy, were evaluated, guided by the RE-AIM evaluation framework. The effectiveness of the intervention (percent screened among participants who were reached) was similar in both arms of the study. This may be due to the fact that CHAs in both arms of the study received the same initial training and used identical program protocols 
Table 2 | Baseline characteristics of participants, all between 50 and 75 years of age and non-adherent to colorectal cancer screening guidelines

\begin{tabular}{|c|c|c|c|c|c|}
\hline & $\begin{array}{l}\text { Basic } \\
\text { implementation } \\
\text { strategy } \\
(N=250)\end{array}$ & $\begin{array}{l}\text { Enhanced } \\
\text { implementation } \\
\text { strategy } \\
(N=423)\end{array}$ & $\begin{array}{c}\text { Total } \\
(N=673)\end{array}$ & $\begin{array}{l}P \\
\text { value }\end{array}$ & $\begin{array}{c}\text { CHIS } \\
(N=226)\end{array}$ \\
\hline $\begin{array}{l}\text { Age (years), mean } \pm \text { SD } \\
\text { (missing } n=10 \text { ) }\end{array}$ & $61 \pm 7$ & $61 \pm 7$ & $61 \pm 7$ & NS & $59 \pm 7$ \\
\hline $\begin{array}{l}\text { Length of US residency } \\
\text { (years), mean } \pm \text { SD } \\
\text { (missing } n=23 \text { ) }\end{array}$ & $19 \pm 12$ & $16 \pm 12$ & $17 \pm 12$ & $<0.001$ & $\begin{array}{l}67 \% \text { lived for } \\
\geq 15 \text { years in } \\
\text { USA }\end{array}$ \\
\hline \multicolumn{6}{|l|}{ Gender } \\
\hline Male & $39 \%$ & $37 \%$ & $38 \%$ & NS & $40 \%$ \\
\hline Female & $61 \%$ & $63 \%$ & $62 \%$ & & $60 \%$ \\
\hline \multicolumn{6}{|l|}{$\begin{array}{l}\text { Marital status } \\
\text { (missing } n=6 \text { ) }\end{array}$} \\
\hline Not married/living-in & $35 \%$ & $37 \%$ & $36 \%$ & NS & $30 \%$ \\
\hline Married/living-in & $65 \%$ & $63 \%$ & $64 \%$ & & $70 \%$ \\
\hline \multicolumn{6}{|l|}{$\begin{array}{l}\text { Annual household } \\
\text { income } \\
\text { (missing } n=95 \text { ) }\end{array}$} \\
\hline Less than $\$ 20 \mathrm{~K}$ & $45 \%$ & $61 \%$ & $55 \%$ & $<0.001$ & $17 \%$ \\
\hline$\$ 20 \mathrm{~K}$ or more & $55 \%$ & $39 \%$ & $45 \%$ & & $83 \%$ \\
\hline \multicolumn{6}{|l|}{$\begin{array}{l}\text { Highest level of } \\
\text { education } \\
\text { (missing } n=2)\end{array}$} \\
\hline Until some college & $31 \%$ & $35 \%$ & $33 \%$ & NS & $45 \%$ \\
\hline College graduate & $59 \%$ & $53 \%$ & $55 \%$ & & $45 \%$ \\
\hline Post-graduate & $10 \%$ & $12 \%$ & $11 \%$ & & $10 \%$ \\
\hline \multicolumn{6}{|l|}{$\begin{array}{l}\text { Country of birth } \\
\text { (missing } n=19 \text { ) }\end{array}$} \\
\hline Philippines & $99 \%$ & $\sim 100 \%$ & $99 \%$ & NS & $77 \%$ \\
\hline USA or other & $1 \%$ & $<1 \%$ & $1 \%$ & & $23 \%$ \\
\hline \multicolumn{6}{|l|}{$\begin{array}{l}\text { Language with friends } \\
\text { (missing } n=5 \text { ) }\end{array}$} \\
\hline Mostly Filipino & $19 \%$ & $24 \%$ & $22 \%$ & NS & $10 \%$ \\
\hline $\begin{array}{l}\text { Equally/mostly } \\
\text { English }\end{array}$ & $81 \%$ & $76 \%$ & $78 \%$ & & $90 \%$ \\
\hline \multicolumn{6}{|l|}{$\begin{array}{l}\text { Consider self } \\
\text { (missing } n=8 \text { ) }\end{array}$} \\
\hline $\begin{array}{l}\text { More Filipino than } \\
\text { American }\end{array}$ & $60 \%$ & $56 \%$ & $57 \%$ & NS & $\mathrm{n} / \mathrm{a}$ \\
\hline $\begin{array}{l}\text { Equally/more } \\
\text { American/neither }\end{array}$ & $40 \%$ & $44 \%$ & $43 \%$ & & \\
\hline \multicolumn{6}{|l|}{$\begin{array}{l}\text { Has health insurance } \\
\text { (missing } n=32 \text { ) }\end{array}$} \\
\hline Yes & $64 \%$ & $58 \%$ & $60 \%$ & NS & $87 \%$ \\
\hline No & $36 \%$ & $42 \%$ & $40 \%$ & & $13 \%$ \\
\hline $\begin{array}{l}\text { Ever had a CRC } \\
\text { screening test }\end{array}$ & $16 \%$ & $19 \%$ & $18 \%$ & NS & $\mathrm{n} / \mathrm{a}$ \\
\hline \multicolumn{6}{|l|}{$\begin{array}{l}\text { Is member of } \\
\text { participating } \\
\text { organization } \\
\text { (missing } n=60 \text { ) }\end{array}$} \\
\hline Yes & $37 \%$ & $47 \%$ & $43 \%$ & 0.022 & $\mathrm{n} / \mathrm{a}$ \\
\hline No & $63 \%$ & $53 \%$ & $57 \%$ & & \\
\hline
\end{tabular}

Participants in the two arms were compared using two-sample $t$ tests for continuous variables and chi-square tests for categorical variables

NS not significant (P>0.05), CHIS California Health Interview Survey 2007 and 2009, Filipino Americans age 50-75, non-adherent to CRC screening guidelines

and materials. CHAs in the enhanced arm received additional booster sessions that also included assistance with recruitment strategies. This additional technical assistance during the start-up phase may have been critical for implementation and subsequent reach of the program, as 10 out of 11 organizations in the enhanced arm implemented the program, compared to only 7 out of 11 organizations in the basic arm. This 


\begin{tabular}{|c|c|c|c|c|c|}
\hline Domain & Description & $\begin{array}{l}\text { Basic } \\
\text { strategy }\end{array}$ & $\begin{array}{l}\text { Enhanced } \\
\text { strategy }\end{array}$ & $\begin{array}{l}P \\
\text { value }\end{array}$ & $\begin{array}{l}\text { Effect } \\
\text { size }^{a}\end{array}$ \\
\hline \multicolumn{6}{|c|}{ Organizational-level adoption and implementation } \\
\hline Adoption & $\begin{array}{l}\text { Number of organizations who agree to } \\
\text { implement the program }\end{array}$ & 11 & 11 & \multicolumn{2}{|c|}{ Equal by design } \\
\hline Implementation & $\begin{array}{l}\text { Proportion of adopting organizations who } \\
\text { implemented program }\end{array}$ & $64 \%(7 / 11)$ & $91 \%(10 / 11)$ & $0.13^{\mathrm{b}}$ & 0.68 \\
\hline \multicolumn{6}{|c|}{ CHA-level adoption, implementation, and reach } \\
\hline Adoption & $\begin{array}{l}\text { Number of CHAs trained per implementing } \\
\text { organization: mean } \pm S D\end{array}$ & $5.3 \pm 0.8(37 / 7)$ & $5.1 \pm 0.6(51 / 10)$ & \multicolumn{2}{|c|}{ Similar by design } \\
\hline Implementation & $\begin{array}{l}\text { Proportion of trained CHAs who delivered } \\
\text { the program }\end{array}$ & $81 \%(30 / 37)$ & $78 \%(40 / 51)$ & $0.90^{c}$ & - \\
\hline Reach per $\mathrm{CHA}$ & $\begin{array}{c}\text { Number of individuals reached per } \\
\text { implementing CHA: mean } \pm \text { SD }\end{array}$ & $\begin{array}{l}8.3 \pm 5.2 \\
(250 / 30)\end{array}$ & $\begin{array}{r}10.6 \pm 5.8 \\
(423 / 40)\end{array}$ & $0.21^{\mathrm{c}}$ & 0.41 \\
\hline \multicolumn{6}{|c|}{ Individual-level effectiveness } \\
\hline Effectiveness & $\begin{array}{l}\text { Proportion of enrolled individuals who } \\
\text { obtained CRC screening }\end{array}$ & $49 \%(122 / 250)$ & $53 \%(223 / 423)$ & $0.51^{d}$ & 0.08 \\
\hline \multicolumn{6}{|c|}{ Reach and impact summary measures } \\
\hline $\begin{array}{l}\text { Reach per } \\
\text { organization }\end{array}$ & $\begin{array}{l}\text { Number of individuals reached per } \\
\text { organization: mean } \pm S D \text { (min, max) }\end{array}$ & $23 \pm 21(0,50)$ & $38 \pm 17(0,49)$ & $0.06^{\mathrm{e}}$ & 0.83 \\
\hline $\begin{array}{l}\text { Impact per } \\
\text { organization } \\
\end{array}$ & $\begin{array}{l}\text { Number of individuals screened per } \\
\text { organization: mean } \pm \text { SD (min, max) }\end{array}$ & $11 \pm 14(0,41)$ & $20 \pm 15(0,45)$ & $0.12^{\mathrm{e}}$ & 0.66 \\
\hline Total reach & $\begin{array}{l}\text { Total number of individuals who received } \\
\text { the program (enrolled) }\end{array}$ & 250 & 423 & & \\
\hline Total impact & Total number of individuals screened & 122 & 223 & & \\
\hline \multicolumn{6}{|c|}{$\begin{array}{l}\text { a Effect sizes were computed as standardized effect sizes (difference in means divided by SD) for continuous variables and } h \text { effect sizes (based on arcs } \\
\text { transformation) for proportions }\end{array}$} \\
\hline \multicolumn{6}{|l|}{${ }^{\mathrm{b}}$ Fisher exact test } \\
\hline \multicolumn{6}{|c|}{${ }^{\mathrm{C}}$ Mixed models with $\mathrm{CHA}$ as unit of analysis } \\
\hline \multicolumn{6}{|c|}{${ }^{\mathrm{d}}$ Mixed logistic regression model with individual as unit of analysis } \\
\hline \multicolumn{6}{|c|}{${ }^{\mathrm{e}}$ Linear regression with organization as unit of analysis } \\
\hline
\end{tabular}

suggests that at least some of the community organizations require ongoing technical assistance during the start-up phase of a program in addition to initial training and distribution of program materials.

Other capacity building components that engaged the leaders of the organizations that were unique to the enhanced arm may also have contributed to the increased reach of the program in this arm. Engagement of the leadership increased the visibility of the program and the support for the program within the organization, including the CHA recruitment efforts. An alternative reason for the increased reach in the enhanced arm is that the additional capacity building activities could have been perceived by the organizations as monitoring of intervention implementation. Several reviews have found that studies that monitored implementation obtained better outcomes than studies that did not monitor implementation [36, 37].

Using RE-AIM metrics to analyze program outcomes and plotting these outcomes as suggested by Glasgow and colleagues [33, 38] illustrate the importance of both reach and effectiveness for estimating the public health impact of the implementation strategies that were tested. Compared to other studies [20, 22], our study assisted a relatively large number of participants in obtaining CRC screening, and given their low income and low level of health insurance, many of our participants would probably have remained unscreened if they had not participated in our study. Our multi-component intervention was quite intensive and achieved a relatively high screening rate (around $50 \%$ ), similar to high rates achieved in two other CRC screening promotion programs among Korean and Vietnamese Americans in non-clinical settings [18, 20]. However, these relatively high screening rates at follow-up may be partially due to selection bias, as explained below.

Program effectiveness in this implementation trial (CRC2 study) compared to our previous effectiveness trial (CRC1 study)-In our initial CRC1 study, we developed and tested the CRC screening promotion intervention, which was delivered by research staff and Filipino American health professionals, and achieved a $30 \%$ screening rate in the intervention group compared to a $9 \%$ screening rate in the control group [6]. In the implementation trial described here (CRC2 study), the program was delivered by trained CHAs instead of research staff and health professionals, although a few of the CHAs had a professional background in health or education. Although the intervention components were the same or very similar in both trials, including the offer of free processing of FOBT kits for participants without insurance, we observed a substantially greater screening rate about $50 \%$ in the implementation trial. This goes against the expectation of "voltage drop, the phenomenon in which interventions 

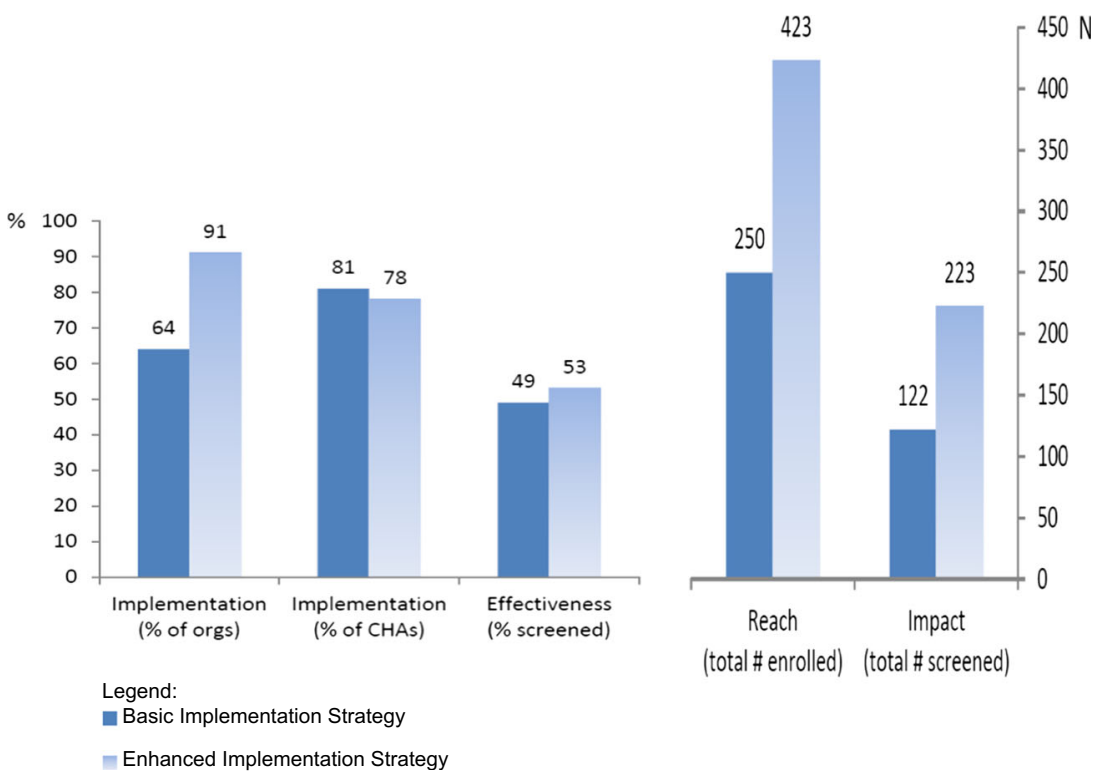

Enhanced Implementation Strategy

Fig. 2 | Performance of two implementation strategies on RE-AIM measures

are expected to yield lower benefits as they move from efficacy to effectiveness and into real world use" [39]. The substantially higher effectiveness of the program compared with our previous trial could be due to several reasons: (1) CHAs typically knew the participants that they recruited and these social bonds may have influenced participants to get screened; (2) CHAs may have recruited those participants who in their view were most likely to complete screening if given the information and the free screening test (selection bias); (3) unlike in the previous study that included a single reminder letter to participants to get screened, CHAs were able to issue personal reminders multiple times by phone or when they met participants at the community organization; (4) participants may have perceived the program to be delivered by their organization rather than an outside academic institution and therefore may have felt more obligated to complete the screening test; and (5) the modification to the study protocol pertaining to the referral of participants to the free clinic even if they had health insurance may have reduced barriers to screening among insured participants. All of these facts may have contributed to the increased effectiveness of the program compared to the initial effectiveness trial. Our trial closely monitored the implementation process in order to better understand it [9]. However, assessments may influence the implementers "on the ground" and may result in a high level of implementation that may not be typical and hard to maintain.

Programs in health care settings versus non-health care settings-Most programs to promote CRC screening are delivered in clinical settings which has a number of advantages: CRC screening fits the mission of a health care setting; a trained workforce of health professionals is available for program implementation; and, if successful, the program may have a good chance of getting incorporated into the health care system, thereby increasing sustainability. The disadvantage is that clinic-based programs typically do not reach individuals who do not see a health care provider, or who only see a provider if they are sick, not for routine screening. Many of the CRC promotion programs in clinical settings have been successful in increasing CRC screening rates among patients who were non-adherent to CRC screening guidelines and have achieved screening rates at 6-month follow-up among these patients ranging from $22 \%$ after an automated reminder telephone call [40] to $65 \%$ and higher after more intensive multi-component interventions $[17,41]$.

Fewer programs have been tested in non-health care settings such as churches and senior centers [6, 20, 22]. These sites can reach individuals who do not have a regular health care provider, and in fact, $40 \%$ of the participants in our study reported that they did not have health insurance. Our implementation trial succeeded in reaching Filipino Americans who have low levels of income and lack of health insurance-two characteristics that have been shown to be associated with lack of CRC screening among Filipino Americans and other ethnic groups [42-44]. Several of these community-based trials have shown good intervention effect sizes and programs in community settings may have promise for reducing disparities in CRC 
screening between the general population and minority groups.

However, implementation of a CRC screening promotion program in non-clinical settings has two important drawbacks: (1) although these programs can increase awareness of the screening tests, reduce barriers to screening, and urge participants to discuss CRC screening with their provider or refer participants without health insurance to a low-cost clinic, individuals still have to go to a health care provider to obtain screening or to submit an FOBT test that they may have received through a program or health fair at a non-clinical setting; and (2) sustainability may pose a problem since community sites may lack funding to maintain the program. In addition, turnover of program staff and organizational leaders may require additional trainings and capacity building activities to maintain program activities.

This study had been powered to detect a moderate difference in effectiveness between arms, as measured by participant CRC screening rates. However, taking a public health perspective, impact, which concerns the total number of individuals screened, may be a more important outcome to compare across strategies. Future implementation studies could consider powering on impact endpoints.

Limitations and conclusions-Our data suggest that the initial implementation of an evidence-based program to promote CRC screening through CHAs was successful in a variety of community settings, achieving a screening rate of around $50 \%$ among Filipino Americans who were not up to date with CRC screening. A more intensive strategy that included more contacts with CHAs and additional capacity building activities with organizational leaders achieved similar screening rates but reached more participants, resulting in a greater public health impact. Community sites received substantial technical and financial assistance during the study period for both program implementation and research activities which was discontinued as the study ended. This may affect the sustainability of the program. CHAs were asked to collect a large amount of information from participants and to keep detailed log sheets about their activities to facilitate the evaluation of the two strategies. Even if some sites were able to continue promotion of CRC screening among their members to some extent, it is unlikely that they would be able to continue such an extensive data collection effort. Some of the organizations, especially some faith-based organizations that have health ministries, may be able to continue some program activities. Future studies should examine what resources would be required for community organizations to sustain programs activities.

Acknowledgments: This work was supported by grant RSGT-04-210-05CPPB from the American Cancer Society and by the UCLA Kaiser Permanente Center for Health Equity. CMC was also supported by NIH/NCI grant P30 CA16042 and AEM by U54-CA143931. We would like to thank the members of the Filipino American community who participated in this study.
Compliance with ethical standards:

Conflicts of interest: Annette Maxwell, Leda Danao, Reggie Cayetano, Catherine Crespi, and Roshan Bastani declare that they have no conflict of interest.

Ethical approval: All procedures performed in studies involving human participants were in accordance with the ethical standards of the institutional and/or national research committee and with the 1964 Helsinki declaration and its later amendments or comparable ethical standards.

1. Colditz GA. The promise and challenges of dissemination and implementation research. In: Brownson RC, Colditz GA, Proctor EK, eds. Dissemination and implementation research in health. New York: Oxford University Press, Inc; 2012: 3-22.

2. Hoeffel EM, Rastogi S, Kim MO, et al. The Asian Population: 2010. 2010 Census Brief issued March 2012. Washington, DC: US Census Bureau; 2012.

3. Maxwell AE, Crespi CM. Trends in colorectal cancer screening utilization among ethnic groups in California: are we closing the gap? Cancer Epidemiol Biomarkers Prev. 2009; 18(3): 752-759.

4. Kandula NR, Wen M, Jacobs EA, et al. Low rates of colorectal, cervical, and breast cancer screening in Asian Americans compared with non-Hispanic whites: cultural influences or access to care? Cancer. 2006; 107(1): 184-192.

5. Lin SS, Clarke CA, Prehn AW, et al. Survival differences among Asian subpopulations in the United States after prostate, colorectal, breast, and cervical carcinomas. Cancer. 2002; 94(4): 1175-1182.

6. Maxwell AE, Bastani R, Danao LL, et al. Results of a communitybased randomized trial to increase colorectal cancer screening among Filipino Americans. Am J Public Health. 2010; 100(11): 2228-2234.

7. Baron RC, Rimer BK, Breslow RA, et al. Client-directed interventions to increase community demand for breast, cervical, and colorectal cancer screening a systematic review. Am J Prev Med. 2008; 35(1 Suppl): S34-S55.

8. Rabin BA, Brownson RC. Developing the terminology for dissemination and implementation research. In: Brownson RC, Colditz GA, Proctor EK, eds. Dissemination and implementation research in health. New York: Oxford University Press; 2012: 23-51.

9. Proctor EK, Brownson RC. Measurement issues in dissemination and implementation research. In: Brownson RC, Colditz GA, Proctor $\mathrm{EK}$, eds. Dissemination and implementation research in health. New York: Oxford University Press; 2012: 261-280.

10. Potter MB, Somkin CP, Ackerson LM, et al. The FLU-FIT program: an effective colorectal cancer screening program for high volume flu shot clinics. Am J Manag Care. 2011; 17(8): 577-583.

11. Potter MB, Walsh JM, Yu TM, et al. The effectiveness of the FLU-FOBT program in primary care a randomized trial. Am J Prev Med. 2011; 41(1): 9-16.

12. Potter MB, Yu TM, Gildengorin G, et al. Adaptation of the FLU-FOBT Program for a primary care clinic serving a low-income Chinese American community: new evidence of effectiveness. J Health Care Poor Underserved. 2011; 22(1): 284-295.

13. Percac-Lima S, Grant RW, Green AR, et al. A culturally tailored navigator program for colorectal cancer screening in a community health center: a randomized, controlled trial. J Gen Intern Med. 2009; 24(2): 211-217.

14. Tu SP, Chun A, Yasui Y, et al. Adaptation of an evidence-based intervention to promote colorectal cancer screening: a quasiexperimental study. Implement Sci. 2014; 9: 85.

15. Thompson NJ, Boyko EJ, Dominitz JA, et al. A randomized controlled trial of a clinic-based support staff intervention to increase the rate of fecal occult blood test ordering. Prev Med. 2000; 30(3): 244-251.

16. Lasser KE, Murillo J, Medlin E, et al. A multilevel intervention to promote colorectal cancer screening among community health center patients: results of a pilot study. BMC Fam Pract. 2009; 10: 37.

17. Green BB, Wang CY, Anderson ML, et al. An automated intervention with stepped increases in support to increase uptake of colorectal cancer screening: a randomized trial. Ann Intern Med. 2013; 158(5 Pt 1): 301-311.

18. Nguyen $\Pi$, Love MB, Liang C, et al. A pilot study of lay health worker outreach and colorectal cancer screening among Chinese Americans. J Cancer Educ. 2010; 25(3): 405-412.

19. Maxwell AE, Danao LL, Bastani R. Dissemination of colorectal cancer screening by Filipino American Community Health 
Advisors: a feasibility study. Health Promot Pract. 2013; 14(4): 498-505.

20. Ma GX, Shive S, Tan Y, et al. Community-based colorectal cancer intervention in underserved Korean Americans. Cancer Epidemiol. 2009; 33(5): 381-386.

21. Nguyen BH, McPhee SJ, Stewart SL, et al. Effectiveness of a controlled trial to promote colorectal cancer screening in Vietnamese Americans. Am J Public Health. 2010; 100(5): 870-876.

22. Blumenthal DS, Smith SA, Majett CD, et al. A trial of 3 interventions to promote colorectal cancer screening in African Americans. Cancer. 2010; 116(4): 922-929.

23. Navarro AM, Raman R, McNicholas LJ, et al. Diffusion of cancer education information through a Latino community health advisor program. Prev Med. 2007; 45(2-3): 135-138.

24. Nelson A, Lewy R, Dovydaitis T, et al. Promotores as researchers: expanding the promotor role in community-based research. Health Promot Pract. 2011; 12(5): 681-688.

25. Messias DK, Parra-Medina D, Sharpe PA, et al. Promotoras de Salud: roles, responsibilities, and contributions in a multisite communitybased randomized controlled trial. Hisp Health Care Int. 2013; 11(2): 62-71.

26. Hill A, De Zapien JG, Staten LK, et al. From program to policy: expanding the role of community coalitions. Prevent Chronic Dis. 2007; 4(4): A103.

27. Maxwell AE, Danao LL, Cayetano RT, et al. Adoption of an evidencebased colorectal cancer screening promotion program by community organizations serving Filipino Americans. BMC Public Health. 2014; 14(1): 246.

28. Hayes RJ, Moulton LH. Cluster randomised trials. Boca Raton, FL: Chapman \& Hall/CRC; 2009.

29. Maxwell AE, Danao LL, Cayetano RT, et al. Evaluating the training of Filipino American community health advisors to disseminate colorectal cancer screening. J Community Health. 2012; 37(6): 12181225.

30. Kessler R, Glasgow RE. A proposal to speed translation of healthcare research into practice: dramatic change is needed. Am J Prev Med. 2011; 40(6): 637-644.

31. Gaglio B, Phillips SM, Heurtin-Roberts S, et al. How pragmatic is it? Lessons learned using PRECIS and RE-AIM for determining pragmatic characteristics of research. Implement Sci. 2014; 9: 96.
32. Glasgow RE, Vogt TM, Boles SM. Evaluating the public health impact of health promotion interventions: the RE-AIM framework. Am J Public Health. 1999; 89(9): 1322-1327.

33. Glasgow RE, Klesges LM, Dzewaltowski DA, et al. Evaluating the impact of health promotion programs: using the RE-AIM framework to form summary measures for decision making involving complex issues. Health Educ Res. 2006; 21(5): 688-694.

34. Cohen J. Statistical power analysis for the behavioral sciences. 2nd ed. Hillsdale, NJ: Lawrence Erlbaum Associated; 1988.

35. Donner A, Klar N. Design and analysis of cluster randomized trials in health research. New York, NY: Oxford University Press; 2000.

36. DuBois DL, Holloway BE, Valentine JC, et al. Effectiveness of mentoring programs for youth: a meta-analytic review. Am J Community Psychol. 2002; 30(2): 157-197.

37. Smith PK, Ananiadou K, Cowie H. Interventions to reduce school bullying. Can J Psychiatry. 2003; 48(9): 591-599.

38. Glasgow RE, Nelson CC, Strycker LA, et al. Using RE-AIM metrics to evaluate diabetes self-management support interventions. $\mathrm{Am}$ J Prev Med. 2006; 30(1): 67-73.

39. Chambers DA, Glasgow RE, Stange KC. The dynamic sustainability framework: addressing the paradox of sustainment amid ongoing change. Implement Sci. 2013; 8: 117.

40. Mosen DM, Feldstein AC, Perrin N, et al. Automated telephone calls improved completion of fecal occult blood testing. Med Care. 2010; 48(7): 604-610.

41. Tu SP, Taylor V, Yasui Y, et al. Promoting culturally appropriate colorectal cancer screening through a health educator: a randomized controlled trial. Cancer. 2006; 107(5): 959-966.

42. Maxwell AE, Danao LL, Crespi CM, et al. Disparities in the receipt of fecal occult blood test versus endoscopy among Filipino American immigrants. Cancer Epidemiol Biomarkers Prev. 2008; 17(8): 19631967.

43. Maxwell AE, Crespi CM, Antonio CM, et al. Explaining disparities in colorectal cancer screening among five Asian ethnic groups: a population-based study in California. BMC Cancer. 2010; 10: 214.

44. Klabunde CN, Brown M, Ballard-Barbash R, et al. Cancer screening-United States, 2010. MMWR Morb Mortal Wkly Rep. 2012; 61(3): 41-45. 